\title{
Screening of core genes and pathways in breast cancer development via comprehensive analysis of multi gene expression datasets
}

\author{
JIE BAI $^{1}$, XIAOYU ZHANG ${ }^{1}$, XIAONING KANG ${ }^{2}$, LIJUN JIN ${ }^{1}$, PENG WANG $^{1}$ and ZUNYI WANG ${ }^{1}$ \\ Departments of ${ }^{1}$ Thyroid and Breast III, and ${ }^{2}$ Ultrasound II, Cangzhou Central Hospital, Cangzhou, Hebei 061001, P.R. China
}

Received August 10,2018; Accepted August 13, 2019

DOI: $10.3892 / 01.2019 .10979$

\begin{abstract}
Breast cancer has been the leading cause of cancer-associated mortality in women worldwide. Perturbation of oncogene and tumor suppressor gene expression is generally considered as the fundamental cause of cancer initiation and progression. In the present study, three gene expression datasets containing information of breast cancer and adjacent normal tissues that were detected using traditional gene microarrays were downloaded and batch effects were removed with $\mathrm{R}$ programming software. The differentially expressed genes between breast cancer and normal tissue groups were closely associated with cancer development pathways. Interestingly, five pathways, including 'extracellular matrix-receptor interaction', 'peroxisome proliferator-activated receptors signaling pathway', 'propanoate metabolism', 'pyruvate metabolism' and 'regulation of lipolysis in adipocytes', were thoroughly connected by 10 genes. Patients with upregulation of six of these hub genes (acetyl-CoA carboxylase $\beta$, acyl-CoA dehydrogenase medium chain, adiponectin, C1Q and collagen domain containing, acyl-CoA synthetase short chain family member 2 , phosphoenolpyruvate carboxykinase 1 and perilipin 1) exhibited improved breast cancer prognosis. Additionally, breast cancer-specific network analysis identified several gene-gene interaction modules. These gene clusters had strong interactions according to the scoring in the whole network, which may be important to the development of breast cancer. In conclusion, the present study may improve the understanding of the mechanisms of breast cancer and provide several valuable prognosis and treatment signatures.
\end{abstract}

\section{Introduction}

Breast cancer is one of the most common types of malignant tumor in women worldwide (1). With $>2$ million new cases

Correspondence to: Dr Zunyi Wang, Department of Thyroid and Breast III, Cangzhou Central Hospital, 16 Xinhuaxi Road, Cangzhou, Hebei 061001, P.R. China

E-mail: wangzunyi18202@outlook.com

Key words: breast cancer, pathway, prognosis, network, survival diagnosed in 2018 worldwide, the incidence rate of breast cancer is increasing year by year (2). According to the statistics of 2014, China accounts for $12.2 \%$ of newly diagnosed cases and $9.6 \%$ of deaths worldwide (3). The survival time of breast cancer has been prolonged with the development of early detection and modern treatment technology (4). At present, the prognosis of breast cancer is mainly evaluated by clinicopathological characteristics, including as age, tumor size, lymph node metastasis and histological grade (5). Breast cancer is a highly heterogeneous tumor at the molecular level. Based on the expression levels of estrogen receptor (ER), progesterone receptor, HER 2 and $\mathrm{Ki}-67$ protein, breast cancer is classified into Luminal A, Luminal B, HER2 overexpression and Basal-like types (6-8). The genetic heterogeneity of breast cancer leads to different therapeutic effects and prognosis of patients with the same pathological type and clinical stage under identical clinical treatment $(9,10)$. Therefore, large-scale genomic studies on the pathogenesis and prognosis of breast cancer have become a hotspot (11). Screening genes for early diagnosis, genotyping and prognosis of breast cancer by gene chip technology is of great significance for guiding individualized treatment and improving prognosis (12).

Breast cancer is a multigenic disease with a multifactorial etiology, and the occurrence of breast cancer is a complicated multistep process $(13,14)$, in which numerous signaling pathways are altered to some extent. Her-2, as an important prognostic factor of breast cancer, is vital for the Ras signaling pathway (15). McGlynn et al (16) used immunohistochemistry to detect the activation of MAPK pathway and revealed the patients had lower activation of MAPK pathway after receiving more effective chemotherapy and endocrine therapy. Wnt signaling pathway, involved in the development of early embryonic mammary gland, leads to the occurrence of breast cancer when abnormally activated (17). Shao et al (18) found that blocking the expression of $\beta$-catenin in Wnt signaling pathway could induce apoptosis of breast cancer cells. Although much progress has been made in this area, the molecular pathogenesis of breast cancer remains not fully understood. The majority of studies use only one set of mRNA expression chip data from either experimental studies (19) or databases (20) to perform analysis of breast cancer, which may have an impact on the analysis results. The present study integrated data from multiple chip platforms and eliminated batch effect for preprocessing by using various function packages 
of R. In addition, pathway and biological target analysis was performed, and may identify areas for the prevention, clinical treatment and prognosis of breast cancer.

\section{Materials and methods}

Gene expression profiles. Gene expression profiles were selected from the Gene Expression Omnibus (GEO) database (https://www.ncbi.nlm.nih.gov/geo) using the following criteria: i) The samples were breast cancer and normal tissues from Homo sapiens; ii) gene expression levels were detected using the Affymetrix Human Genome U133 Plus 2.0 Array (GEO ref. no. GPL570; Thermo Fisher Scientific, Inc.). A total of three datasets (accession nos. GSE29431 (21), GSE42568 (22) and GSE61304 $(23,24)$ ) were downloaded. The sample characteristics of the three datasets are shown in Table I.

Gene expression preprocessing. To remove the effect induced by biological replicates within a specific dataset and to correct the batch effect among different datasets, intra- and inter-group normalization were performed using R Bioconductor (version 3.6; http://www.bioconductor.org), Affymetrix microarray analysis (25) and surrogate variable analysis (sva) packages (26), respectively. The affy $\mathrm{R}$ package was applied to each specific dataset for normalization followed by background correction using a robust multi-array (RMA) method (27) and $\log _{2}$-transformation. The sva package was designed for the removal of batch effects and other unwanted variation in high-throughput experiments, such as the experimental conditions, through identifying and estimating surrogate variables for unknown sources, removing known batch effects using the ComBat function of sva package (28) or removing batch effects with known control probes. In the present study, the sva package was used to remove all potential batch effects among the three datasets. Probe IDs were then annotated to gene symbols according to GPL570 annotation information. The expression values of genes annotated by more than one probe ID were summarized.

Differential expression analysis. The expression datasets were combined, and the expression matrix consisting of 216 breast cancer tissue samples and 33 normal tissue samples was obtained. The differentially expressed genes (DEGs) in breast cancer samples compared with normal tissue samples were then identified using the $\mathrm{R}$ Bioconductor limma package (version 3.4.0; https://bioconductor. org/packages/release/bioc/html/limma.html) (29) with the thresholds of fold change $>2$ (upregulated) or $<0.5$ (downregulated) and false discovery rate $<0.05$.

Enrichment analysis. Kyoto Encyclopedia of Genes and Genomes (KEGG) pathways potentially involved in breast cancer initiation and progression were identified using the $\mathrm{R}$ Bioconductor clusterProfiler package (version 3.10) (30) with a threshold of $\mathrm{P}<0.05$. Additionally, the associations between significantly enriched pathways and expression profile alterations of the genes in these pathways were explored using the $\mathrm{R}$ Bioconductor enrichplot package (version 1.4; https://github. com/GuangchuangYu/enrichplot). Gene Ontology (GO)
Table I. Sample characteristics of the three gene expression datasets used in the present study.

\begin{tabular}{lccc}
\hline & & \multicolumn{2}{c}{ Characteristic } \\
\cline { 3 - 4 } GEO ID & $\begin{array}{c}\text { Sample } \\
\text { size, } \mathrm{n}\end{array}$ & $\begin{array}{c}\text { Tumor tissue, } \\
\mathrm{n} \text { (case) }\end{array}$ & $\begin{array}{c}\text { Breast normal } \\
\text { tissue, } \mathrm{n} \text { (control) }\end{array}$ \\
\hline GSE29431 & 66 & 54 & 12 \\
GSE42568 & 121 & 104 & 17 \\
GSE61304 & 62 & 58 & 4 \\
\hline
\end{tabular}

GEO, Gene Expression Omnibus.

enrichment analysis of differentially expressed genes in breast cancer was performed through the Database for Annotation, Visualization, and Integrated Discovery (DAVID; http://david. ncifcrf.gov/). The Biological Processes (BP) terms were obtained, and the final result was visualized by the enrichMap function in Cytoscape software.

Construction of disease-specific network. The breast cancer-specific network containing the interactions among the DEGs was constructed using the Search Tool for the Retrieval of Interacting Genes/Proteins (STRING) database (version 11.0; https://string-db.org). Scores between 0 and 1 were assigned to the interaction pairs deposited in STRING according to their methods, such as bioinformatics prediction, high-throughput gene microarray and immunoprecipitation. In the present study, only interacting pairs with combined scores $>0.4$ were considered as reliable. Additionally, to highlight the network modules that represented stronger interactions among genes from the whole network, module analysis was applied to the breast cancer-specific network via the enrichmentMap and MCODE plug-in for Cytoscape software (version 3.5.1) (31).

Evaluation of the association between hub genes and breast cancer prognosis. Prognosis is an important indicator to assess drug effectiveness and relations between gene expression and disease progression. In the present study, the Kaplan-Meier plotter database (www.kmplot.com), which contains the genome-wide gene expression profiles of $>5,000$ breast cancer samples, was used to evaluate associations between hub genes and breast cancer prognosis. The samples were classified into two groups according to the upper quartile expression value of a specific gene, and overall survival (OS) differences between the high expression and the low expression group were explored using the log-rank test. $\mathrm{P}<0.05$ was considered to indicate a statistically significant difference.

Cell culture, RNA extraction and reverse transcription-quantitative PCR (RT-qPCR). The normal breast epithelial (MCF10A) and breast cancer (MDA-MB-231) cells used in the present study were purchased from the American Type Culture Collection. The cells were cultured at a density of $2 \times 10^{4}$ cells $/ \mathrm{cm}^{2}$ in $100-\mathrm{mm}$ tissue culture dishes (Corning Inc.) with DMEM medium (Solarbio Science \& Technology Co., Ltd.) containing $10 \%(\mathrm{v} / \mathrm{v}) \mathrm{FBS}$ and $100 \mathrm{mg} / \mathrm{ml}$ 
Table II. Primer sequences for quantitative PCR.

\begin{tabular}{|c|c|c|c|}
\hline Gene & Primer sequence $\left(5^{\prime}-3^{\prime}\right)$ & Product length, bp & Temperature, ${ }^{\circ} \mathrm{C}$ \\
\hline \multirow[t]{2}{*}{$\mathrm{ACACB}$} & F: CAAGCCGATCACCAAGAGTAAA & 79 & 59 \\
\hline & R: CCCTGAGTTATCAGAGGCTGG & & \\
\hline \multirow[t]{2}{*}{ ACADM } & F: ACAGGGGTTCAGACTGCTATT & 240 & 58 \\
\hline & R: TCCTCCGTTGGTTATCCACAT & & \\
\hline \multirow[t]{2}{*}{ ACDC } & F: TGCTGGGAGCTGTTCTACTG & 248 & 59 \\
\hline & R: TACTCCGGTTTCACCGATGTC & & \\
\hline \multirow[t]{2}{*}{ ACSS2 } & F: AAAGGAGCAACTACCAACATCTG & 159 & 59 \\
\hline & R: GCTGAACTGACACACTTGGAC & & \\
\hline \multirow[t]{2}{*}{ PCK1 } & F: AAAACGGCCTGAACCTCTCG & 98 & 60 \\
\hline & R: ACACAGCTCAGCGTTATTCTC & & \\
\hline \multirow[t]{2}{*}{ PLIN1 } & F: TGTGCAATGCCTATGAGAAGG & 154 & 59 \\
\hline & R: AGGGCGGGGATCTTTTCCT & & \\
\hline \multirow[t]{2}{*}{$\beta$-actin } & F: AGCGAGCATCCCCCAAAGTT & 285 & 60 \\
\hline & R: GGGCACGAAGGCTCATCATT & & \\
\hline
\end{tabular}

ACACB, acetyl-CoA carboxylase $\beta$; ACADM, acyl-CoA dehydrogenase medium chain; ACDC, adiponectin, C1Q and collagen domain containing; ACSS2, acyl-CoA synthetase short chain family member 2; PCK1, phosphoenolpyruvate carboxykinase 1; PLIN1, perilipin 1; F, forward; R, reverse.

penicillin-streptomycin (all Gibco; Thermo Fisher Scientific, Inc.) at $37^{\circ} \mathrm{C}$ in a humidified incubator with $5 \% \mathrm{CO}_{2}$. All cells were passaged once after reaching confluence with $0.25 \%$ trypsin-EDTA solution (Sigma-Aldrich; Merck KGaA).

Total RNA was extracted from MCF10A and MDA-MB-231 cells using TRIzol ${ }^{\circledR}$ reagent (Invitrogen; Thermo Fisher Scientific, Inc.) according to the manufacturer's protocol. A NanoDrop-1000 spectrophotometer (Thermo Fisher Scientific, Inc.) was used to quantify RNA by measuring the optical density, and the purity was assessed by determining the OD260/OD280 ratio and samples with a ratio between 1.9 and 2.0 were considered to be pure. The RNA samples were reverse transcribed to cDNA using SuperScript ${ }^{\mathrm{TM}}$ IV First-Strand Synthesis System (Invitrogen; Thermo Fisher Scientific, Inc.), with the temperature protocol of $25^{\circ} \mathrm{C}$ for $10 \mathrm{~min}, 42^{\circ} \mathrm{C}$ for $50 \mathrm{~min}, 70^{\circ} \mathrm{C}$ for $15 \mathrm{~min}$, and $4^{\circ} \mathrm{C}$ for cooling. The cDNA samples were diluted in diethyl pyrocarbonate-treated water at a ratio of 1:5. qPCR was then performed using a LightCycler 480 SYBR Green I master kit (Roche Applied Science) for 38 cycles of $95^{\circ} \mathrm{C}$ for $30 \mathrm{sec}, 58^{\circ} \mathrm{C}$ for $50 \mathrm{sec}$, and $72^{\circ} \mathrm{C}$ for 1 min. $\beta$-actin was used as an endogenous control gene, and all primers used are listed in Table II. All samples were run in triplicate on the ABI 7900HT Real-Time PCR system (Applied Biosystems; Thermo Fisher Scientific, Inc.). Calculation of relative expression levels was performed using the $2^{-\Delta \Delta q}$ method (32).

Statistical analysis. The data for survival analysis was downloaded from The Cancer Genome Atlas (TCGA) dataset (www.cancergenome.nih.gov). The survival analysis and Kaplan-Meier curves plotting were conducted by using survival and survminer packages in R language. All data are expressed as the mean \pm standard deviation unless otherwise indicated. There were five samples per group included in order to obtain the statistical results. Comparisons between multiple groups were evaluated using one-way ANOVA, followed by Tukey's honestly significant difference test in GraphPad Prism version 5.0 software (GraphPad Software, Inc.). $\mathrm{P}<0.05$ was considered to indicate a statistically significant difference.

\section{Results}

DEGs. The expression value distributions before and after correcting the batch effect for the combined datasets are presented in Fig. 1A-D, respectively. The density plot of the gene expression distribution (Fig. 1A and C) indicated that the differences in expression value between breast cancer and normal tissue samples were magnified by batch effect correction. Additionally, the quantile-quantile (Q-Q) plots (Fig. 1B and D) revealed that the distance between dots and the normal distribution line became closer after the batch effect was removed. Therefore, the gene expression datasets after normalization should have been reliable for the subsequent analysis. Differential expression analysis identified a total of 1,110 DEGs (335 upregulated and 775 downregulated) in breast cancer samples compared with normal tissue samples. The mean expression values of those genes in the control and tumor samples across the three datasets are shown in Table SI.

Significantly enriched KEGG pathways. As shown in Fig. 2A, 22 KEGG pathways were enriched with the DEGs identified following the batch effect correction. The top five KEGG pathways included 'focal adhesion', 'cAMP signaling pathway', 'peroxisome proliferator-activated receptors (PPAR) signaling pathway', 'relaxin signaling pathway', and 'extracellular matrix (ECM)-receptor interaction'. The intersecting KEGG pathways were visualized according to overlapping gene sets. As shown in Fig. 2B, five KEGG pathways were connected by 10 genes, 
A

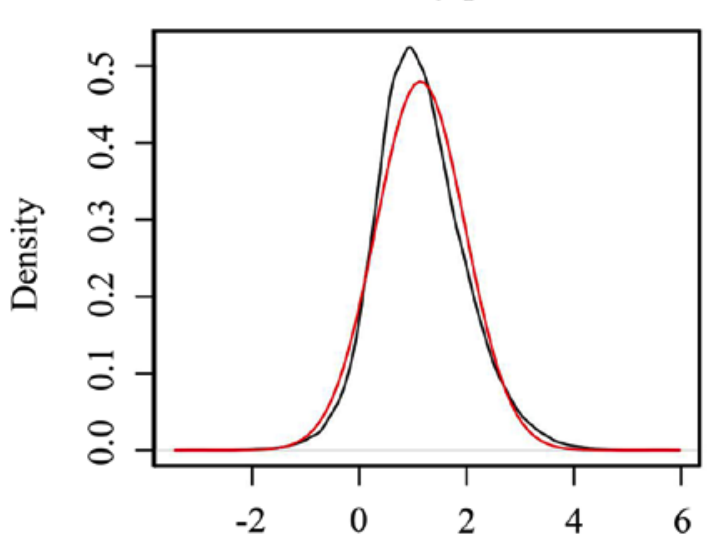

$\mathrm{N}=20545 \quad$ Bandwidth $=0.09709$
B

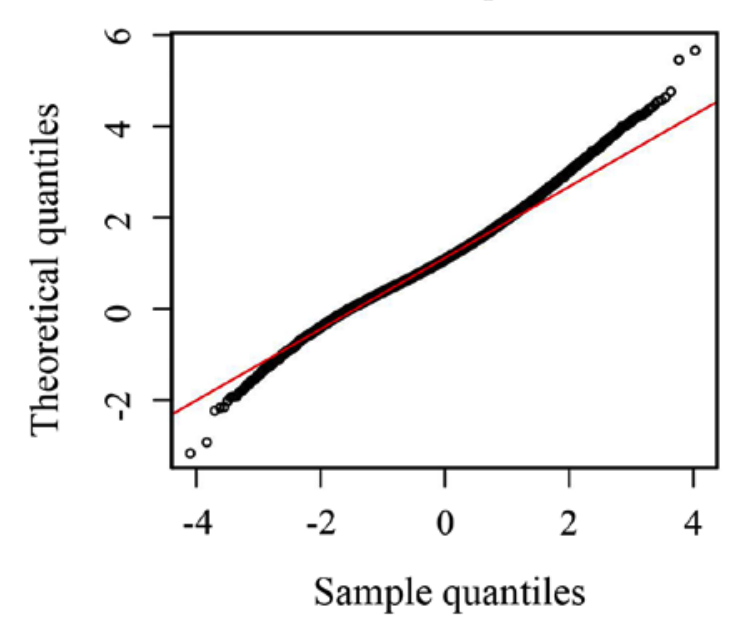

$\mathrm{C}$

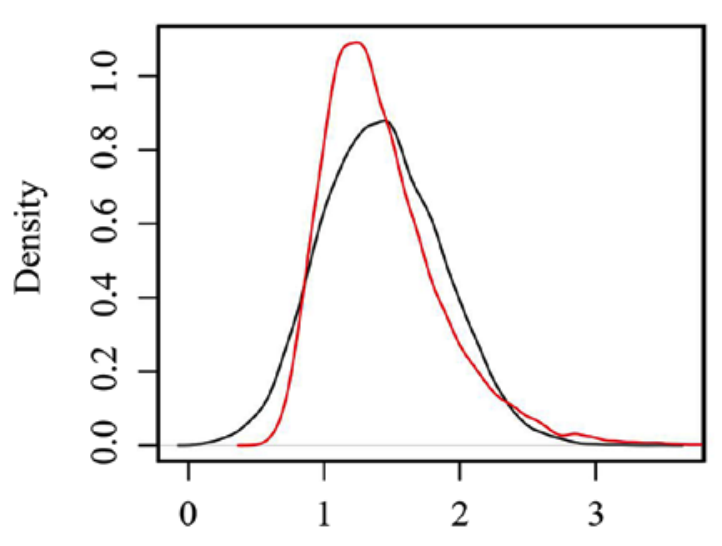

$\mathrm{N}=20545 \quad$ Bandwidth $=0.05484$
D

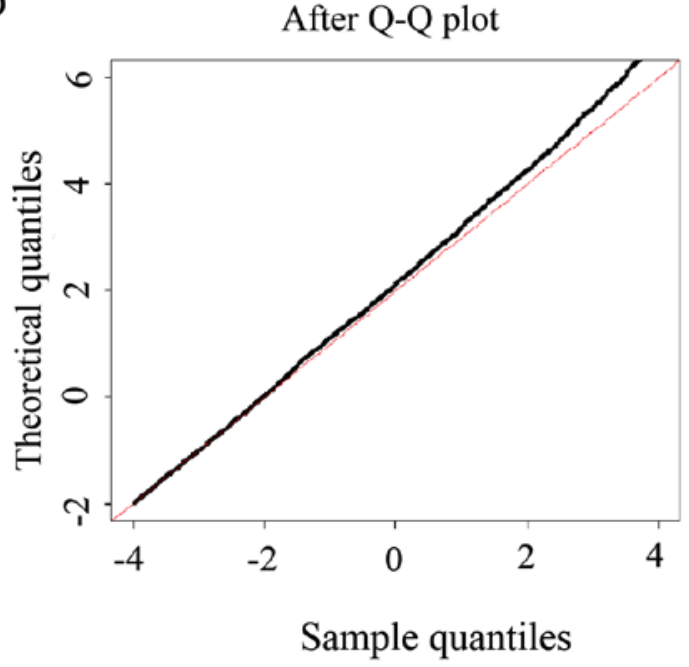

Figure 1. The density and QQ plots for evaluating the effect of the intergroup normalization method on overall expression (A) The density plot before batch effect removal. (B) The QQ plot before batch effect removal. (C) The density plot after batch effect removal. (D) The QQ plot after batch effect removal. N represented the gene numbers covered by gene microarray. The red line represented breast cancer samples, and the black line represents paracancer samples.

including CD36, enoyl-CoA hydratase and 3-hydroxyacyl CoA dehydrogenase (EHHADH), acyl-CoA dehydrogenase medium chain (ACADM), malic enzyme 1 (ME1), phosphoenolpyruvate carboxykinase 1 (PCK1), fatty acid binding protein 4 (FABP4), perilipin 1 (PLIN1), acyl-CoA synthetase short chain family member 2 (ACSS2), lactate dehydrogenase B (LDHB) and acetyl-CoA carboxylase $\beta$ (ACACB). These 10 genes were considered to be hub genes, and all of them were downregulated (shown as green nodes) in breast cancer samples. The core of the intersected KEGG pathways was the 'PPAR signaling pathway'. Additionally, the majority of the genes contained in 'propanoate metabolism' (9/10), 'pyruvate metabolism' (11/12) and 'regulation of lipolysis in adipocytes' (13/15) were downregulated (shown as green nodes) in breast cancer samples, whereas more upregulated (red nodes) genes were contained in the 'ECM-receptor interaction pathway' (7/19).

Gene Ontology (GO) is another important gene functions analysis method that could intuitively uncover biological processes, molecular functions and cellular component terms closely associated with a list of genes. A total of four GO term clusters were identified by GO enrichment analysis combined with crosstalk analysis. The clusters were mainly associated with biological process regulation, substance metabolism, cell cycle and response to stimulus (Fig. 2C).

Associations between hub gene expression and breast cancer $O S$. In the present study, genes shared by at least two pathways were considered to serve potential roles in breast cancer development and considered to be hub genes. The association between the expression levels of the10 genes and the survival rate of breast cancer was investigated by Kaplan-Meier survival analysis. The results indicated that increased expression levels of ACACB, ACADM, adiponectin, $\mathrm{C} 1 \mathrm{Q}$ and collagen domain containing (ACDC), ACSS2, PCK1 and PLIN1 were significantly associated with improved breast cancer prognosis (Fig. 3), which illustrates their potential as tumor suppressor genes. Tables SII and SIII illustrate the associations between these hub genes and common breast cancer clinicopathologic features, including age, sex, ethnicity and survival status in addition to Tumor-Node-Metastasis 

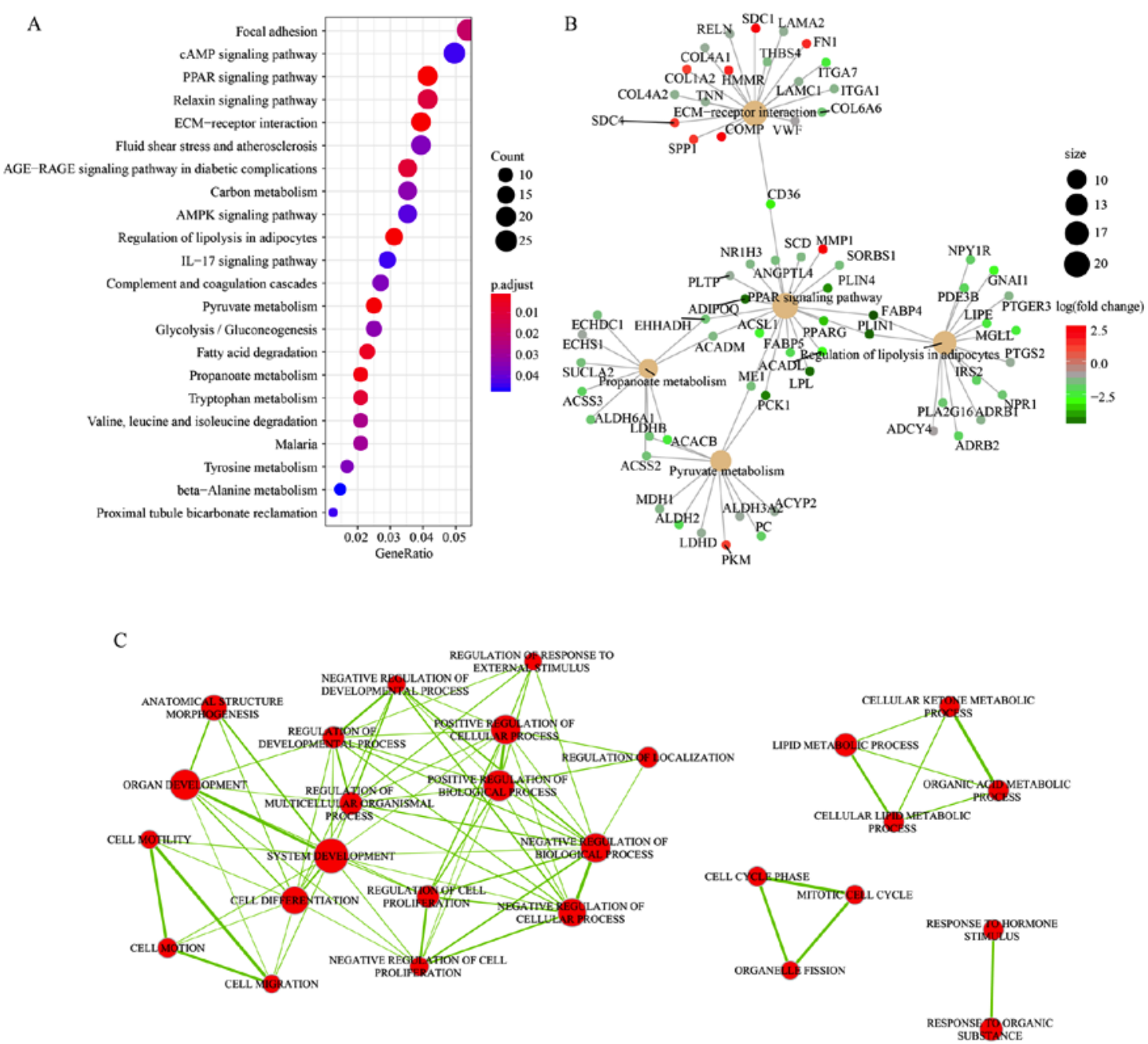

Figure 2. Functional enrichment analysis of differentially expressed genes. (A) Significantly enriched KEGG pathways. Circle size and color indicate the gene number contained in a specific pathway and their significance, respectively. (B) Associations among KEGG pathways represented by their shared genes. Larger pathway node size indicates more genes contained in the pathway, and color gradient from green to red indicates downregulation to upregulation of the corresponding gene. (C) Crosstalk analysis of significantly enriched Gene Ontology terms. Two nodes are connected if there are any shared genes between them, and a thicker line represents more shared genes. KEGG, Kyoto Encyclopedia of Genes and Genomes.

(TNM) stage (33). RT-qPCR was performed to validate the expression levels of ACACB, ACADM, ACDC, ACSS2, PCK1 and PLIN1 in breast cancer MDA-MB-231 and normal breast epithelial MCF10A cell lines. As shown in Fig. 4, the results revealed that the expression levels of the six hub genes were all downregulated in the MDA-MB-231 cells compared with in the MCF10A cells. Among them, the differences for ACACB, ACSS2, PCK1 and PLIN1 were all significant.

Subsequently, the prognosis values of the six hub genes were validated based on the TCGA-BRCA dataset (Fig. S1). In addition, Kaplan-Meier analysis for those six hub genes in triple-negative breast cancer (TNBC) samples indicated the same impact of genes ACACB, ACADM and ACSS2, whose increased expression significantly associated with worse TNBC prognosis, on TNBC prognosis (Fig. S2).

Breast cancer-specific network. Among the 1,110 DEGs that were identified, a total of 4,937 interaction pairs were identified by the STRING database with a threshold of combined score $>0.4$. Additionally, module analysis was further applied to the whole network for network interpretability by using the MCODE plug-in in Cytoscape. As a result, a total of four modules were obtained by MCODE, which represented the sub-networks, as presented in Fig. 5 with green and red nodes representing downregulated and upregulated genes in breast cancer, respectively.

\section{Discussion}

Breast cancer is associated with alterations in a number of growth factors and hormone-regulated signaling pathways (34). Clinical data demonstrated that the breast epithelial cells in high estrogen concentration would increase the risk of breast cancer (35). The expression of vascular endothelial growth factor (VEGF) is enhanced under hypoxic conditions inside the tumor, and the neovascular wall promotes the metastasis of tumor cells (36). Poor prognosis of different subtypes is the result of alterations in distinct signaling pathways or transcriptional programs (37). The luminal A subtype has the longest disease-free survival, due to the low mutation rate of TP53 and sensitivity to endocrine therapy (3). The prognosis of 

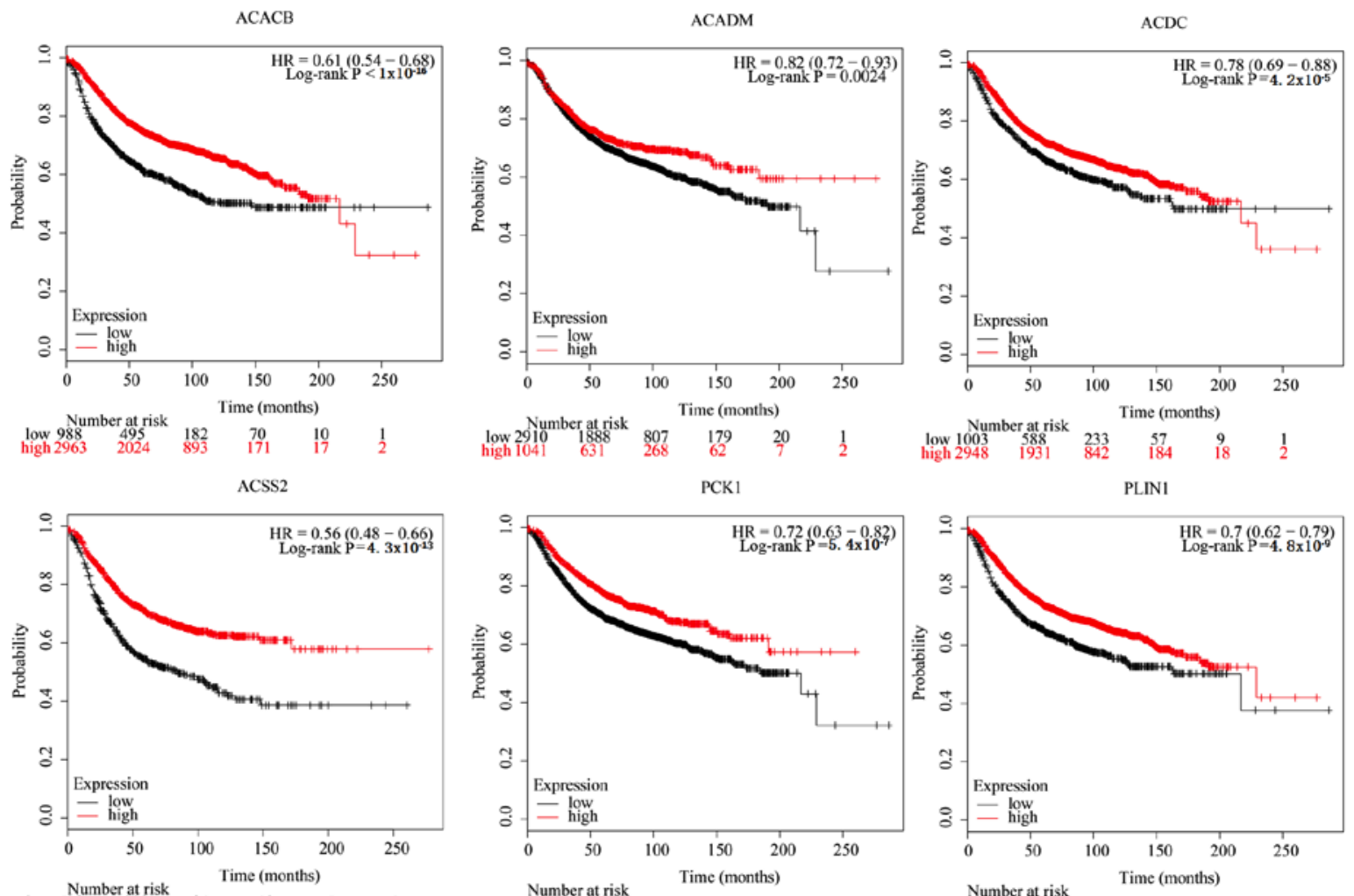

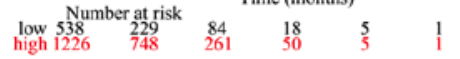

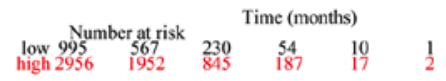

Figure 3. Kaplan-Meier survival curves of samples in the Kaplan-Meier plotter database stratified by expression values of ACACB, ACADM, ACDC, ACSS2, PCK1 and PLIN1. HR, hazard ratio; ACACB, acetyl-CoA carboxylase $\beta$; ACADM, acyl-CoA dehydrogenase medium chain; ACDC, adiponectin, C1Q and collagen domain containing; ACSS2, acyl-CoA synthetase short chain family member 2; PCK1, phosphoenolpyruvate carboxykinase 1; PLIN1, perilipin 1.
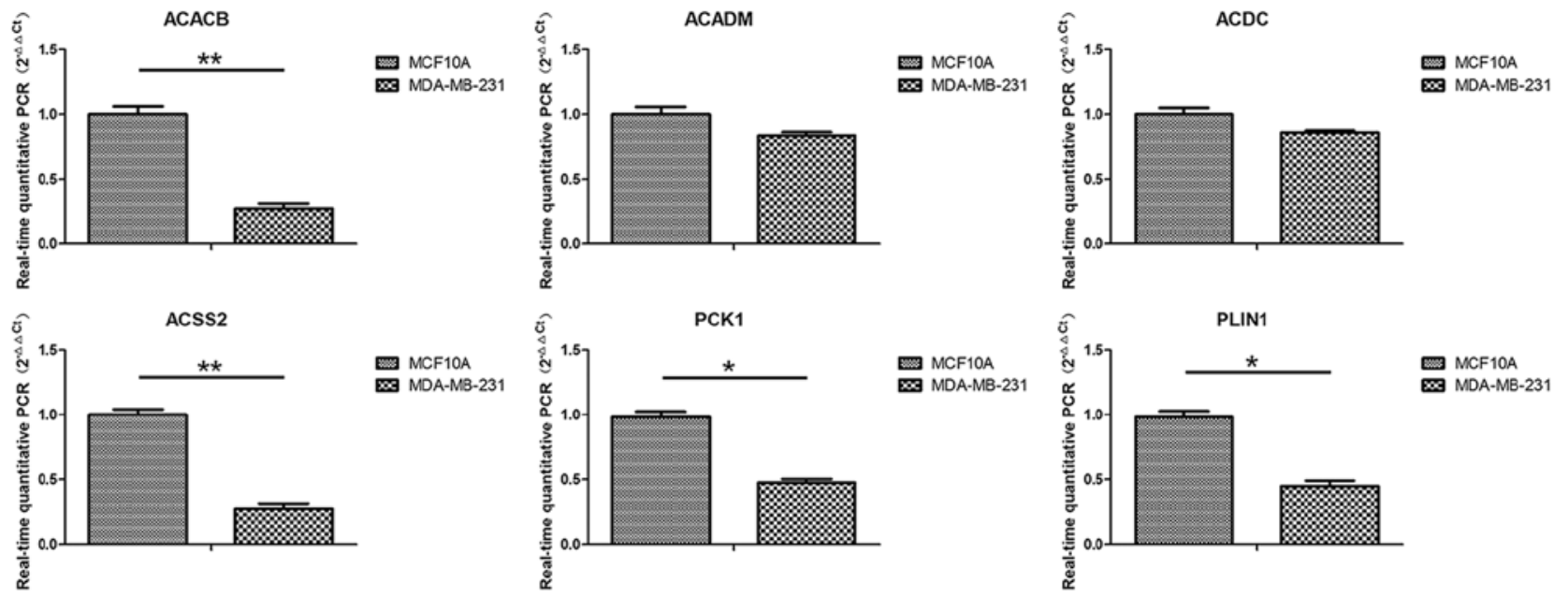

Figure 4. Expression levels of hub genes (ACACB, ACADM, ACDC, ACSS2, PCK1 and PLIN1) in normal and tumor cell lines were evaluated using RT-qPCR The expression levels were measured and normalized to $\beta$-actin. The gene expression levels of MDA-MB-231 cells were compared with those of MCF10A cells. The data are presented as the mean $\pm \mathrm{SD}, \mathrm{n}=3 .{ }^{*} \mathrm{P}<0.05 ;{ }^{* *} \mathrm{P}<0.01$, as indicated. ACACB, acetyl-CoA carboxylase $\beta$; ACADM, acyl-CoA dehydrogenase medium chain; ACDC, adiponectin, C1Q and collagen domain containing; ACSS2, acyl-CoA synthetase short chain family member 2; PCK1, phosphoenolpyruvate carboxykinase 1; PLIN1, perilipin 1; RT-qPCR, real-time-quantitative PCR.

the luminal B subtype is worse than that of the luminal A type, which may be associated with the positive expression of Her-2 receptor and the high expression of Ki67 (38). The poor prognosis of Her-2 subtype is due to the upregulation of epidermal growth factor receptor, which promotes the proliferation and metastasis of cancer cells (38). The high mutation rate of TP53 and overexpression of EGFR in
TNBC (tri-negative breast cancer) subtype results in lower tumor differentiation and poorest prognosis (39). In the past decade, gene microarray and immunohistochemistry techniques have been applied to explore the molecular typing and prognosis prediction of breast cancer. The 21-gene Oncotype (40), 70-gene MammaPrint signature (41) and 76-gene expression profile (42) have high ability to predict 
A

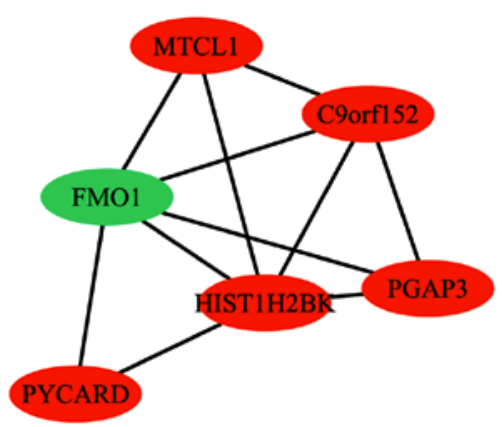

B

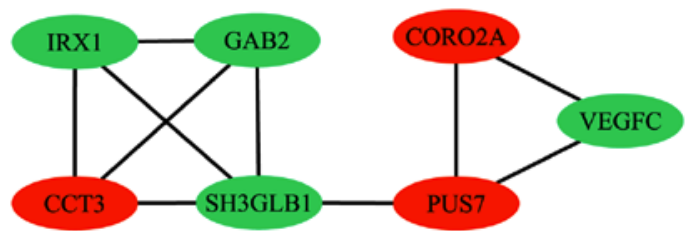

$\mathrm{C}$

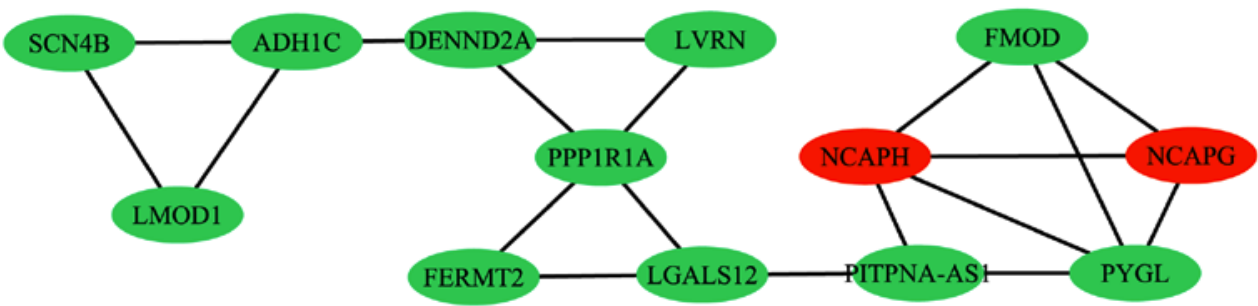

$\mathrm{D}$

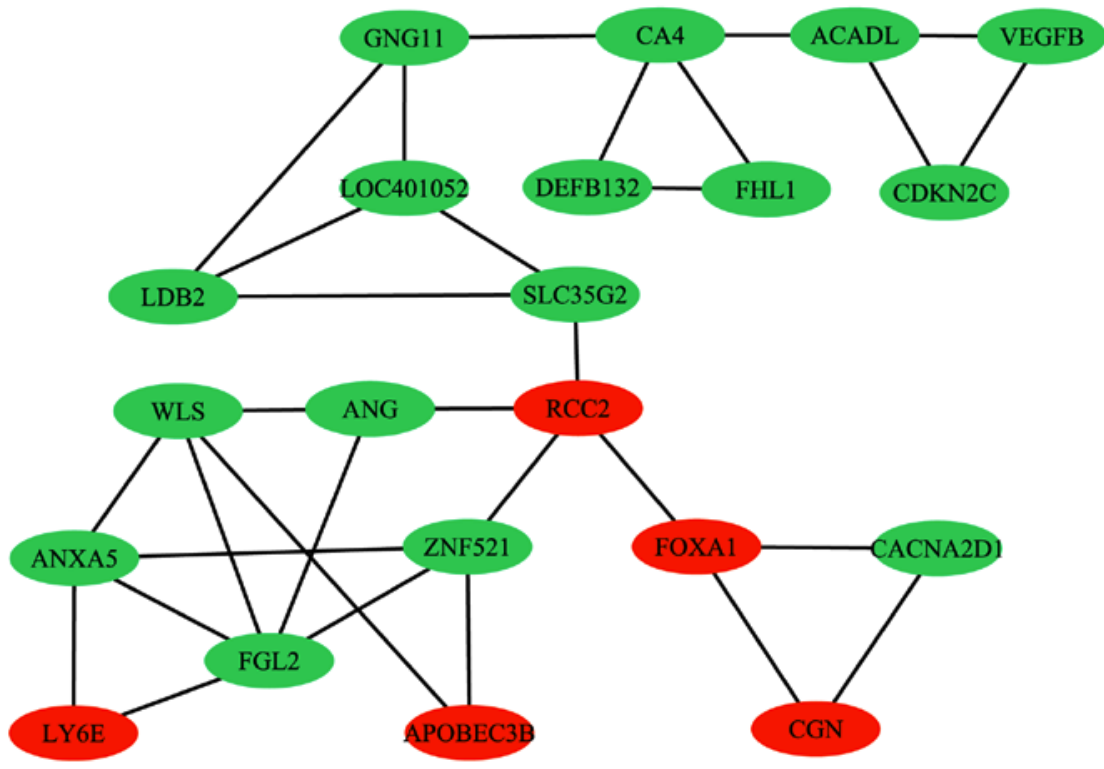

Figure 5. Module analysis of breast cancer-specific network. (Breast cancer-specific network modules obtained through MCODE analysis of the whole network, namely the subnetworks. Panels A, B, C and D represented the subnetworks respectively. Red and green nodes indicate upregulation and downregulation in breast cancer samples, respectively.

the prognosis of patients with breast cancer. Combined with traditional pathological types and clinical data they provide useful guidance for clinical treatment. Since currently the gene expression profiles that are available from different databases have their own limitations, such as samples from patients that were limited to one clinic or geographical region, or errors may occur between different platforms, research on prognostic genes for breast cancer is continuing in order to identify more accurate and widely applicable prognostic genes.

In the present study, the results of the analysis of breast cancer from 249 samples, including 216 tumor and 33 normal tissue samples, were described. The present study differed from previously reported gene expression profiles, and their data were presented from different microarray platforms, for example 48/70 genes in a study by Hartmann et al (41) were identified on the Affymetrix U133a array while 38/76 genes in a study by Wang et al (42) were identified on the Agilent array. The data from the three datasets included in the present study were all obtained from the same microarray platform (Affymetrix U133a array) and R bioconductor was used to eliminate the batch effect. KEGG pathway enrichment analysis of DEGs in breast cancer revealed that 'focal adhesion pathway', 'PPAR signaling pathway', 'ECM-receptor interaction', 'regulation of lipolysis in adipocytes', 'pyruvate metabolism' and 'propanoate metabolism pathway' were significantly enriched. Focal adhesion kinase is a central regulator of focal adhesion, influencing cell proliferation and 
migration $(43,44)$. Focal adhesions serve as mechanical links to the ECM and signaling center of cell communication, and have been implicated in tumor invasion (45). The findings of the present study were consistent with research that suggests early tumor cell migration and invasion of neighboring tissues are mediated by focal adhesion signaling (46). PPARs are nuclear hormone receptors that are activated by fatty acids and their derivatives (47). The findings also indicated that the PPAR signaling pathway may be an important predictor of breast cancer response to neoadjuvant chemotherapy (48). Adipocytes are a major component of breast tissue. Obesity is associated with increased recurrence and reduced survival of breast cancer. Increasing evidence suggests that obesity leads to larger breast tumor size, high risk of distant metastasis and increased mortality $(49,50)$. Breast tumor cells exposed to adipocyte-conditioned media or in coculture with adipocytes exhibit the ability to alter proliferation, migration and invasion (51-53). Similar effects have also been observed in 3-D cultures and xenograft models $(53,54)$. The enrichment analysis results provided genes that may be involved in lipolysis in adipocytes on tumor cells and may lead to the development of novel cancer control strategies.

A gene concept network was constructed and revealed that the five significant pathways, 'PPAR signaling pathway', 'ECM-receptor interaction', 'propanoate metabolism', 'pyruvate metabolism', and 'regulation of lipolysis in adipocytes' identified from the KEGG analysis were linked by 10 downregulated genes, including CD36, EHHADH, ACADM, ME1, PCK1, FABP4, PLIN1, ACSS2, LDHB and ACACB. Survival analysis demonstrated that the expression levels of ACACB, ACADM, ACDC, ACSS2, PCK1 and PLIN1 were significantly positively associated with the survival of patients with breast cancer. Notably, the low expression gene ACACB screened in the present study is also included in the 76-gene expression profile (42). ACC2, encoded by the ACACB gene, serves an important role in the oxidation of fatty acids (55). Low expression levels of ACACB indicate an increase in fatty acid oxidation (56). In a previous study that evaluated changes in expression of several selected genes, patients with high levels of ACACB had better prognosis following neoadjuvant chemotherapy, and with the exception of ER-patients, ACACB adds independent prognostic value in multivariable models including all 24 genes in the 126 patients, as well as the ER+/HER2-patients (57). Inhibition of ACC2 reduces proliferation and de novo lipogenesis of tumor cells $(58,59)$. In addition, inhibition of ACC2 rewires cancer metabolism and enables head and neck squamous cell carcinoma cells to survive inhibition of the Warburg effect by addition of cetuximab (60). The present analysis demonstrated that ACACB was downregulated in breast cancer and positively associated with survival time thus supported previous studies that inhibition of fatty acid synthesis may be a promising target to reduce drug resistance of tumor cells.

As presented in Fig. 2A, with the exception of various cancer-associated pathways, such as tyrosine metabolism, the Ras signaling pathway occurs multiple times. Ras is a small guanosine triphosphate-binding protein that serves an important role in signal transduction pathways that influence cellular proliferation, apoptosis, cytoskeletal organization and other important biological processes (61). Ras mutations lead to constitutive activation of the Ras signaling pathway in certain human cancer types (62). The majority of patients with colorectal cancer have codon 12 and 13 mutations of K-Ras, which occur in the early stage of the development of cancer (63). While Ras genes are not commonly mutated in human breast cancer, this signaling pathway can be activated by mutations within associated genes, including tyrosine kinase receptors, such as HER2, as well as kinases downstream of Ras, such as mitogen-activated protein kinase (MAPK) or extracellular regulated protein kinase (ERK) (64). Despite large genomic surveys such as The Cancer Genome Atlas demonstrating infrequent canonical mutations in this signaling pathway, several studies (65-67) support targeting the Ras/mitogen-activated protein kinase cell signaling pathway in breast cancer.

In the present study, by selecting samples and removing the batch effect of different datasets, pathway and biological target analysis was performed to obtain several candidate genes that may be involved in breast cancer progression and reoccurrence. Gene prognostic models can provide more accurate prognostic evaluation than clinicopathological indicators, and thus provide a more important reference value for the selection of individualized treatment options. However, since the dataset used did not provide information regarding the breast cancer types, the pathogenesis mechanism associated with the molecular characteristics of different cancer types requires further analysis.

\section{Acknowledgements}

Not applicable.

\section{Funding}

No funding was received.

\section{Availability of data and materials}

The datasets generated and/or analyzed during the current study are available in the GEO repository, https://www.ncbi.nlm.nih. gov/geo/query/acc.cgi?acc=GSE29431, https://www.ncbi.nlm. nih.gov/geo/query/acc.cgi?acc=GSE42568 and https://www. ncbi.nlm.nih.gov/geo/query/acc.cgi?acc=GSE61304.

\section{Authors' contributions}

JB contributed to the design of the study, wrote the manuscript and analyzed the data. XZ and XK revised the manuscript and contributed to the design of the study. LJ and PW acquired, analyzed and interpreted the data. ZW made substantial contributions to the conception and design of the present study, and revised the manuscript. All authors read and approved the final manuscript.

\section{Ethics approval and consent to participate}

Not applicable.

\section{Patient consent for publication}

Not applicable. 


\section{Competing interests}

The authors declare that they have no competing interests.

\section{References}

1. Siegel RL, Miller KD and Jemal A: Cancer statistics, 2018. CA Cancer J Clin 68: 7-30, 2018.

2. Bray F, Ferlay J, Soerjomataram I, Siegel RL, Torre LA and Jemal A: Global cancer statistics 2018: GLOBOCAN estimates of incidence and mortality worldwide for 36 cancers in 185 countries. CA Cancer J Clin 68: 394-424, 2018.

3. Fan L, Strasser-Weippl K, Li JJ, St Louis J, Finkelstein DM, Yu KD, Chen WQ, Shao ZM and Goss PE: Breast cancer in China. Lancet Oncol 15: e279-e289, 2014.

4. Al-Mubarak M, Sacher AG, Ocana A, Vera-Badillo F, Seruga B and Amir E: Fulvestrant for advanced breast cancer: A meta-analysis. Cancer Treat Rev 39: 753-758, 2013.

5. Qiu AF, Miao ZL, Ge GK, Wang CB, Bian J, Ma HY and Xu Q: Response and prognosis of neoadjuvant dose-dense or standard schedule chemotherapy with anthracyclines and taxanes for Luminal B breast cancer. Zhonghua Yi Xue Za Zhi 97: 3466-3470, 2017.

6. Perou CM, Sørlie T, Eisen MB, van de Rijn M, Jeffrey SS, Rees CA, Pollack JR, Ross DT, Johnsen H, Akslen LA, et al: Molecular portraits of human breast tumors. Nature 406: 747-752, 2000.

7. Sorlie T, Tibshirani R, Parker J, Hastie T, Marron JS, Nobel A, Deng S, Johnsen H, Pesich R, Geisler S, et al: Repeated observation of breast tumor subtypes in independent gene expression data sets. Proc Natl Acad Sci USA 100: 8418-8423, 2003.

8. Hu Z, Fan C, Oh DS, Marron JS, He X, Qaqish BF, Livasy C Carey LA, Reynolds E, Dressler L, et al: The molecular portraits of breast tumors are conserved across microarray platforms. BMC Genomics 7: 96, 2006.

9. Kumar M, Sahu RK, Goyal A, Sharma S, Kaur N, Mehrotra R, Singh UR and Hedau S: BRCA1 promoter methylation and expression-associations with ER+, PR+ and HER2+ subtypes of breast carcinoma. Asian Pac J Cancer Prev 18: 3293-3299, 2017.

10. Meisel J, Zhang C, Neely C, Mendoza P, You S, Han T, Liu Y, Sahin AA, O'Regan R and Li X: Evaluation of prognosis in hormone receptor-positive/HER2-negative and lymph node-negative breast cancer with low oncotype DX recurrence score. Clin Breast Cancer 18: 347-352, 2018.

11. Nik-Zainal S, Davies H, Staaf J, Ramakrishna M, Glodzik D, Zou X, Martincorena I, Alexandrov LB, Martin S, Wedge DC, et al: Landscape of somatic mutations in 560 breast cancer whole-genome sequences. Nature 534: 47-54, 2016.

12. Moldovan L, Mitroi A, Petrescu C and Aschie M: Classification of breast carcinomas according to gene expression profiles. J Med Life 6: 14-17, 2013.

13. Shaposhnikov SA, Akopov SB, Chernov IP, Thomsen PD, Joergensen C, Collins AR, Frengen E and Nikolaev LG: A map of nuclear matrix attachment regions within the breast cancer loss of heterozygosity region on human chromosome16q22.1. Genomics 89: 354-361, 2007.

14. Tryndyak V, Kovalchuk O and Pogribny IP: Identification of differentially methylated sites within unmethylated DNA domains in normal and cancer cells. Anal Biochem 356: 202-207, 2006.

15. Hakim AA, Barry CP, Barnes HJ, Anderson KE, Petitte J, Whitaker R, Lancaster JM, Wenham RM, Carver DK, Turbov J, et al: Ovarian Adenocarcinomas in the Laying Hen and Women Share Similar Alterations in p53, ras and HER-2/neu. Cancer Prev Res (Phila) 2: 114-121, 2009.

16. Mcglynn LM, Kirkegaard T, Edwards J, Tovey S, Cameron D, Twelves C, Bartlett JM and Cooke TG: Ras/Raf-1/MAPK pathway mediates response to tamoxifen but not chemotherapy in breast cancer patients. Clin Cancer Res 15: 1487-1495, 2009.

17. Boras-Granic $\mathrm{K}$ and Wysolmerski JJ: Wnt signaling in breast organogenesis. Organogenesis 4: 116-122, 2008.

18. Shao H, Ma J, Guo T and Hu R: Triptolide induces apoptosis of breast cancer cells via a mechanism associated with the Wnt/ß-catenin signaling pathway. Exp Ther Med 8: 505-508, 2014.

19. Klintman M, Buus R, Cheang MC, Sheri A, Smith IE and Dowsett M: Changes in expression of genes representing key biologic processes after neoadjuvant chemotherapy in breast cancer, and prognostic implications in residual disease. Clin Cancer Res 22: 2405-2416, 2016.
20. Liu F, Wu Y, Mi Y, Gu L, Sang M and Geng C: Identification of core genes and potential molecular mechanisms in breast cancer using bioinformatics analysis. Pathol Res Pract 215: 152436, 2019.

21. Cuadros M, Cano C, López FJ, Talavera P, García-Peréz I, Blanco A and Concha A: HER2 status in breast cancer: Experience of a Spanish national reference Centre. Clin Transl Oncol 13: 335-340, 2011.

22. Clarke C, Madden SF, Doolan P, Aherne ST, Joyce H, O'Driscoll L, Gallagher WM, Hennessy BT, Moriarty M, Crown J, et al: Correlating transcriptional networks to breast cancer survival: A large-scale coexpression analysis. Carcinogenesis 34: 2300-2381, 2013.

23. Grinchuk OV, Motakis E, Yenamandra SP, Ow GS, Jenjaroenpun P, Tang Z, Yarmishyn AA, Ivshina AV and Kuznetsov VA: Sense-antisense gene-pairs in breast cancer and associated pathological pathways. Oncotarget 6: 42197-42221, 2015.

24. Aswad L, Yenamandra SP, Ow GS, Grinchuk O, Ivshina AV and Kuznetsov VA: Genome and transcriptome delineation of two major oncogenic pathways governing invasive ductal breast cancer development. Oncotarget 6: 36652-36674, 2015.

25. Linton KM, Hey Y, Saunders E, Jeziorska M, Denton J, Wilson CL, Swindell R, Dibben S, Miller CJ, Pepper SD, et al: Acquisition of biologically relevant gene expression data by Affymetrix microarray analysis of archival formalin-fixed paraffin-embedded tumours. Br J Cancer 98: 1403-1414, 2008.

26. Leek JT and Storey JD: Capturing heterogeneity in gene expression studies by surrogate variable analysis. PLoS Genet 3: 1724-1735, 2007.

27. Irizarry RA, Hobbs B, Collin F, Beazer-Barclay YD, Antonellis KJ, Scherf U and Speed TP: Exploration, normalization, and summaries of high density oligonucleotide array probe level data. Biostatistics 4: 249-264, 2003 .

28. Wang M, Huang J, Liu Y, Ma L, Potash JB and Han S: COMBAT: A combined association test for genes using summary statistics. Genetics 207: 883-891, 2017.

29. Smyth GK: Limma: Linear models for microarray data. Bioinf Computat Biol Solutions Using R Bioconductor: 397-420, 2011.

30. Yu G, Wang LG, Han Y and He QY: clusterProfiler: An R package for comparing biological themes among gene clusters. OMICS 16: 284-287, 2012.

31. Shannon P, Markiel A, Ozier O, Baliga NS, Wang JT, Ramage D, Amin N, Schwikowski B and Ideker T: Cytoscape: A software environment for integrated models of biomolecular interaction networks. Genome Res 13: 2498-2504, 2003.

32. Livak KJ and Schmittgen TD: Analysis of relative gene expression data using real-time quantitative PCR and the 2(-Delta Delta C(T)) method. Methods 25: 402-408, 2001.

33. Belldegrun A, Tsui KH, deKernion JB and Smith RB: Efficacy of nephron-sparing surgery for renal cell carcinoma: Analysis based on the new 1997 tumor-node-metastasis staging system. J Clin Oncol 17: 2868-2875, 1999.

34. Wright KL, Adams JR, Liu JC, Loch AJ, Wong RG, Jo CE, Beck LA, Santhanam DR, Weiss L, Mei X, et al: Ras signaling is a key determinant for metastatic dissemination and poor survival of luminal breast cancer patients. Cancer Res 75: 4960-4972, 2015.

35. Paliouras M, Borgono C and Diamandis EP: Human tissue kallikreins: The cancer biomarker family. Cancer Lett 249: 61-79, 2007.

36. Jin Q, Hemminki K, Enquist K, Lenner P, Grzybowska E, Klaes R, Henriksson R, Chen B, Pamula J, Pekala W, et al: Vascular endothelial growth factor polymorphisms in relation to breast cancer development and prognosis. Clin Cancer Res 11: 3647-3653, 2005 .

37. Gatza ML, Lucas JE, Barry WT, Kim JW, Wang Q, Crawford MD, Datto MB, Kelley M, Mathey-Prevot B, Potti A and Nevins JR: A pathway-based classification of human breast cancer. Proc Natl Acad Sci USA 107: 6994-6999, 2010.

38. Wiechmann L, Sampson M, Stempel M, Jacks LM, Patil SM, King T and Morrow M: Presenting features of breast cancer differ by molecular subtype. Ann Surg Oncol 16: 2705-2710, 2009.

39. Savage K, Leung S, Todd SK, Brown LA, Jones RL, Robertson D, James M, Parry S, Rodrigues Pinilla SM, Huntsman D and Reis-Filho JS: Distribution and significance of caveolin 2 expression in normal breast and invasive breast cancer: An immunofluorescence and immunohistochemical analysis. Breast Cancer Res Treat 110: 245-256, 2008. 
40. Paik S, Shak S, Tang G, Kim C, Baker J, Cronin M, Baehner FL, Walker MG, Watson D, Park T, et al: A multigene assay to predict recurrence of tamoxifen-treated, node-negative breast cancer. N Engl J Med 351: 2817-2826, 2004.

41. Hartmann S, Gerber B, Elling D, Heintze K and Reimer T: The 70-Gene signature as prognostic factor for elderly women with hormone Receptor-Positive, HER2-Negative breast cancer. Breast Care (Basel) 7: 19-24, 2012.

42. Wang Y, Klijn JG, Zhang Y, Sieuwerts AM, Look MP, Yang F, Talantov D, Timmermans M, Meijer-van Gelder ME, Yu J, et al: Gene-expression profiles to predict distant metastasis of lymph-node-negative primary breast cancer. Lancet 365 : 671-679, 2005.

43. Chan KT, Cortesio CL and Huttenlocher A: FAK alters invadopodia and focal adhesion composition and dynamics to regulate breast cancer invasion. J Cell Biol 185: 357-370, 2009.

44. Hiscox S, Barnfather P, Hayes E, Bramble P, Christensen J, Nicholson RI and Barrett-Lee P: Inhibition of focal adhesion kinase suppresses the adverse phenotype of endocrine-resistant breast cancer cells and improves endocrine response in endocrine-sensitive cells. Breast Cancer Res Treat 125: 659-669, 2011.

45. Chen CS, Alonso JL, Ostuni E, Whitesides GM and Ingber DE: Cell shape provides global control of focal adhesion assembly. Biochem Biophys Res Commun 307: 355-361, 2003.

46. Tamura M, Gu J, Takino T and Yamada KM: Tumor suppressor PTEN inhibition of cell invasion, migration, and growth: Differential involvement of focal adhesion kinase and p130Cas Cancer Res 59: 442-449, 1999.

47. Hihi AK, Michalik L and Wahli W: PPARs: Transcriptional effectors of fatty acids and their derivatives. Cell Mol Life Sci 59: 790-798, 2002.

48. Chen YZ, Xue JY, Chen CM, Yang BL, Xu QH, Wu F, Liu F, Ye X, Meng X, Liu GY, et al: PPAR signaling pathway may be an important predictor of breast cancer response to neoadjuvant chemotherapy. Cancer Chemother Pharmacol 70: 637-644, 2012

49. Balaban S, Shearer RF, Lee LS, van Geldermalsen M, Schreuder M, Shtein HC, Cairns R, Thomas KC, Fazakerley DJ, Grewal T, et al: Adipocyte lipolysis links obesity to breast cancer growth: Adipocyte-derived fatty acids drive breast cancer cell proliferation and migration. Cancer Metab 5: 1, 2017.

50. Ligibel JA and Strickler HD: Obesity and its impact on breast cancer: Tumor incidence, recurrence, survival, and possible interventions. Am Soc Clin Oncol Educ Book: 52-59, 2013 doi 10.1200/EdBook_AM.2013.33.52.

51. Dirat B, Bochet L, Dabek M, Daviaud D, Dauvillier S, Majed B, Wang YY, Meulle A, Salles B, Le Gonidec S, et al: Cancer-associated adipocytes exhibit an activated phenotype and contribute to breast cancer invasion. Cancer Res 71: 2455-2465, 2011.

52. Carter JC and Church FC: Mature breast adipocytes promote breast cancer cell motility. Exp Mol Pathol 92: 312-317, 2012.

53. Wang C, Gao C, Meng K, Qiao H and Wang Y: Human adipocytes stimulate invasion of breast cancer MCF-7 cells by secreting IGFBP-2. PLoS One 10: e0119348, 2015.

54. Delort L, Lequeux C, Dubois V, Dubouloz A, Billard H, Mojallal A, Damour O, Vasson MP and Caldefie-Chézet F: Reciprocal interactions between breast tumor and its adipose microenvironment based on a 3D adipose equivalent model. PLoS One 8: e66284, 2013.

55. Riancho JA, Vázquez L, García-Pérez MA, Sainz J, Olmos JM, Hernández JL, Pérez-López J, Amado JA, Zarrabeitia MT, Cano A and Rodríguez-Rey JC: Association of ACACB polymorphisms with obesity and diabetes. Mol Genet Metab 104: 670-676, 2011
56. Ma L, Mondal AK, Murea M, Sharma NK, Tönjes A, Langberg KA, Das SK, Franks PW, Kovacs P, Antinozzi PA, et al: The effect of ACACB cis-variants on gene expression and metabolic traits. PLoS One 6: e23860, 2011.

57. Klintman M, Buus R, Cheang MC, Sheri A, Smith IE and Dowsett M: Changes in expression of genes representing key biologic processes after neoadjuvant chemotherapy in breast cancer, and prognostic implications in residual disease. Clin Cancer Res 22: 2405-2416, 2016.

58. Jones JE, Esler WP, Patel R, Lanba A, Vera NB, Pfefferkorn JA and Vernochet C: Inhibition of Acetyl-CoA carboxylase 1 (ACC1) and 2 (ACC2) reduces proliferation and de novo lipogenesis of EGFRviii human glioblastoma cells. PLoS One 12: e0169566, 2017.

59. Svensson RU, Parker SJ, Eichner LJ, Kolar MJ, Wallace M, Brun SN, Lombardo PS, Van Nostrand JL, Hutchins A, Vera L, et al: Inhibition of acetyl-CoA carboxylase suppresses fatty acid synthesis and tumor growth of non-small-cell lung cancer in preclinical models. Nat Med 22: 1108-1119, 2016.

60. Luo J, Hong Y, Lu Y, Qiu S, Chaganty BK, Zhang L, Wang X, Li Q and Fan Z: Acetyl-CoA carboxylase rewires cancer metabolism to allow cancer cells to survive inhibition of the Warburg effect by Cetuximab. Cancer Lett 384: 39-49, 2017.

61. Bifulco M: Role of the isoprenoid pathway in ras transforming activity, cytoskeleton organization, cell proliferation and apoptosis. Life Sci 77: 1740-1749, 2005

62. Kreeger PK, Mandhana R, Alford SK, Haigis KM and Lauffenburger DA: RAS mutations affect tumor necrosis factor-induced apoptosis in colon carcinoma cells via ERK-modulatory negative and positive feedback circuits along with Non-ERK pathway effects. Cancer Res 69: 8191-8199, 2009.

63. Bazan V,Migliavacca M,Zanna I,TubioloC, Grassi N,LatteriMA, La Farina M, Albanese I, Dardanoni G, Salerno S, et al: Specific codon $13 \mathrm{~K}$-ras mutations are predictive of clinical outcome in colorectal cancer patients, whereas codon $12 \mathrm{~K}$-ras mutations are associated with mucinous histotype. Ann Oncol 13: 1438-1446, 2002.

64. Sun T, Aceto N, Meerbrey KL, Kessler JD, Zhou C, Migliaccio I, Nguyen DX, Pavlova NN, Botero M, Huang J, et al: Activation of multiple proto-oncogenic tyrosine kinases in breast cancer via loss of the PTPN12 phosphatase. Cell 144: 703-718, 2011.

65. Li T and Sparano JA: Inhibiting Ras signaling in the therapy of breast cancer. Clin Breast Cancer 3: 405-420, 2003.

66. Del Grosso C, Antoniol G, Merlo E, et al: The impact of Ras/MAPK/S6K signaling pathway on prediction of clinical outcome in metastatic Her-2 positive breast cancer patients treated with trastuzumab. Cancer Res 74 (19 Suppl): Abstract nr LB-181, 2014

67. Giltnane JM and Balko JM: Rationale for targeting the Ras/MAPK pathway in triple-negative breast cancer. Discov Med 17: 275-283, 2014.

This work is licensed under a Creative Commons Attribution-NonCommercial-NoDerivatives 4.0 International (CC BY-NC-ND 4.0) License. 